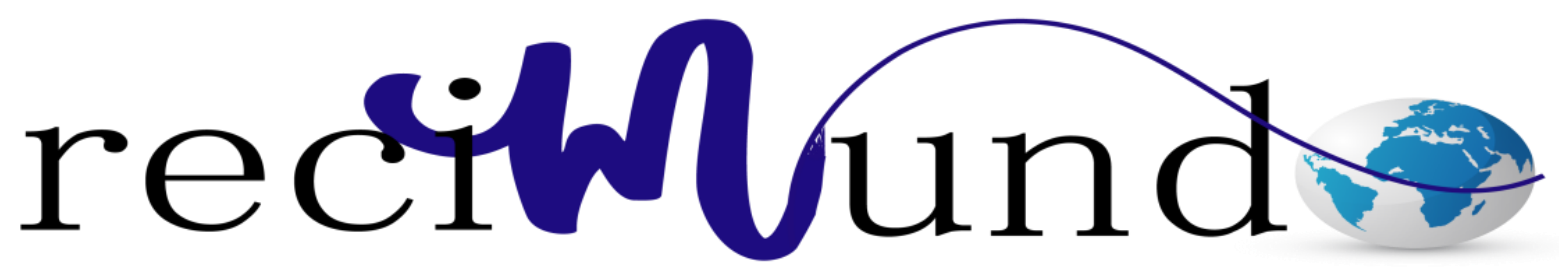

Revista Científica Mundo de la Investigación y el Conocimiento

José Santiago Insuasti Rincones ${ }^{\text {a }}$ J Jossue Tarquino Narvaez Guerrero ${ }^{\text {b; }}$ Vanessa Angelica Chiquito Cedeño ${ }^{c}$; Samuel Wilfrido Prado Moncada ${ }^{d}$

Efectividad de la arginina en el tratamiento de la sensibilidad dentaria

Effectiveness of arginine in the treatment of tooth sensitivity

Revista Científica Mundo de la Investigación y el Conocimiento. Vol. 2 núm.3, julio, ISSN: 2588-073X, 2018, pp. 615-633

DOI: 10.26820/recimundo/2.(3).julio.2018.615-633

Editorial Saberes del Conocimiento

Recibido: 05/04/2018

Aceptado: 03/06/2018

Publicado: 30/07/2018

Correspondencia: jossue.narvaezg@ug.edu.ec

a. Odontólogo de la Universidad de Guayaquil; jose.insuastir@ug.edu.ec

b. Especialista en Periodoncia e Implantologia Quirúrgica; Odontólogo; Universidad de Guayaquil; jossue.narvaezg@ug.edu.ec

c. Odontóloga de la Universidad de Guayaquil; vanessa.chiquitoc@ug.edu.ec

d. Odontólogo de la Universidad de Guayaquil; samuel.pradom@ug.edu.ec 


\section{Efectividad de la arginina en el tratamiento de la sensibilidad dentaria}

Vol. 2, núm. 3., (2018)

José Santiago Insuasti Rincones; Jossue Tarquino Narvaez Guerrero; Vanessa Angelica Chiquito Cedeño; Samuel Wilfrido Prado Moncada

\section{RESUMEN}

La hipersensibilidad dentinaria se define como un dolor corto, agudo, punzante y localizado, como respuesta a estímulos químicos, térmicos, físicos o mecánicos sobre la dentina expuesta. Su etiología es múltiple, sin embargo, se asocia con la pérdida de esmalte. La prevalencia de la hipersensibilidad varía entre un 9\%-55\% de la población según distintas investigaciones la edad promedio es de 20 a 40 años siendo más prevalente en mujeres que en hombres. El objetivo de este estudio es evaluar la eficacia en la reducción de la hipersensibilidad dentinaria utilizando dentífricos que contienen arginina al 8\%. Este estudio clínico, aleatorio, ciego y controlado con dos grupos paralelos, y tres semanas de seguimiento, el cual la muestra de trabajo fue de 30 pacientes donde fueron seleccionados aleatoriamente y se distribuyeron por grupos de sexo y evaluados tras una y tres semanas de uso del dentífricos. Se les aplicó aire proveniente de la jeringa triple del equipo dental para comprobar si existía sensibilidad y observando ciertos factores desencadenantes ante esta anomalía. La cuantificación del dolor se realizó a través de la Escala Visual Análoga (EVA). En la investigación se concluye que el uso de los dentífricos que contienen arginina $8 \%$, reduce de la hipersensibilidad dentinaria tras una y tres semanas de uso de las pastas dentales. Esto también depende de la técnica correcta de cepillado que debe llevar el paciente.

Palabras claves: Sensibilidad dental, arginina, dentífricos, esmalte dental. 


\title{
Efectividad de la arginina en el tratamiento de la sensibilidad dentaria
}

Vol. 2, núm. 3., (2018)

José Santiago Insuasti Rincones; Jossue Tarquino Narvaez Guerrero; Vanessa Angelica Chiquito

Cedeño; Samuel Wilfrido Prado Moncada

\begin{abstract}
Dentin hypersensitivity is defined as a short, sharp, sharp, localized response to chemical, thermal, physical or mechanical stimuli on exposed dentin pain. The aetiology is multiple, however, it is associated with the loss of enamel. The prevalence of hypersensitivity varies between $9 \%-55 \%$ of the population according to various studies the average age is 20 to 40 years being more prevalent in women than in men. The aim of this study is to evaluate the efficacy in reducing dentin hypersensitivity using toothpaste containing $8 \%$ arginine. This clinical study, randomized, blind, controlled trial with two parallel groups, and three weeks of monitoring, which the working sample was 30 patients which were selected randomly and distributed by groups of sex and evaluated after three weeks of use the dentifrices. It was applied triple air from the syringe from dental equipment to see if there was sensitivity and observing certain triggers to this anomaly. Quantification of pain was performed using the Visual Analogue Scale (VAS). In research it is concluded that the use of toothpaste containing $8 \%$ arginine, reduces dentinal hypersensitivity after three weeks of use of toothpastes. This also depends on the correct brushing technique should take the patient.
\end{abstract}

Keywords: Tooth sensitivity, arginine, toothpaste, dental enamel. 


\section{Efectividad de la arginina en el tratamiento de la sensibilidad dentaria}

Vol. 2, núm. 3., (2018)

José Santiago Insuasti Rincones; Jossue Tarquino Narvaez Guerrero; Vanessa Angelica Chiquito Cedeño; Samuel Wilfrido Prado Moncada

\section{Introducción.}

La hipersensibilidad dentinaria se refiere a un dolor corto, agudo, punzante y localizado, como respuesta a estímulos químicos, térmicos, físicos o mecánicos sobre la dentina expuesta. Su etiología es múltiple, se asocia con la pérdida de esmalte (causado por abrasión, atrición, caries, etc.) o debido a la presencia de recesiones gingivales (cepillado traumático, enfermedad periodontal, etc.) (Márquez, Quintero, Sanz, Ramírez, Inostroza \& Chaparro., 2016)

La prevalencia de la hipersensibilidad de acuerdo con Márquez y colaboradores varía entre un 9\%-55\% de la población según distintas investigaciones la edad promedio es de 20 a 40 años. Siendo más prevalente en mujeres que en hombres. En cuanto a su división en la arcada dentaria, provoca daños principalmente las superficies vestibulares de caninos y premolares superiores, luego por incisivos y molares mandibulares (Márquez, Quintero, Sanz, Ramírez, Inostroza \& Chaparro., 2016).

Así mismo produce la pérdida de los tejidos periodontales en pacientes que presenta periodontitis como su terapia mecánica (eliminación de movimiento del biofilm supragingival y subgingival) representan agentes asociados a la aparición de hipersensibilidad dentinaria. Averiguaciones previas reportan entre un 9\%-23\% de hipersensibilidad cervical en forma previa a la terapia convencional (Ardila, 2009).

Según Álvarez y colaboradores la teoría hidrodinámica del dolor consiste en que los fluidos existentes al interior de los túbulos dentinarios se descubriría igualdad osmótica, se encuentran en forma directa con la pulpa dentaria. La aparición de estímulos externos mecánicos, químicos, térmicos, alteran el equilibrio osmótico, afectando los baro-receptores, originando un 


\section{Efectividad de la arginina en el tratamiento de la sensibilidad dentaria}

Vol. 2, núm. 3., (2018)

José Santiago Insuasti Rincones; Jossue Tarquino Narvaez Guerrero; Vanessa Angelica Chiquito Cedeño; Samuel Wilfrido Prado Moncada

impulso nervioso por parte de las fibras nerviosas que están en la pulpa de la pieza dentaria (Alvarez, Arroyo, Aranguiz, Contreras, \& Moncada., 2010).

Últimamente, se ha producido un nuevo producto para el tratamiento de la hipersensibilidad dentinaria, basándose en la terapéutica de cierto componente como: arginina al $8 \%$ y a carbonato de calcio. Según sus fabricantes, la aplicación tópica de este producto en las áreas que presenta sensibilidad dentaria disminuiría significativamente los niveles de dolor y sensibilidad cervical luego de una sola aplicación. (Márquez, Quintero, Sanz, Ramírez, Inostroza \& Chaparro., 2016).

Al respecto la arginina es un aminoácido, con carga positiva, a un pH fisiológico de 6.5 a 7.5 y se obtiene de los alimentos, poseería un efecto beneficioso en la oclusión de los túbulos dentinarios abiertos, por el cual resiste la irritación de ácidos procedentes de la dieta, con cualidades de baja conductancia hidráulica y con una estructura química pequeña en nitrógeno y carbono, pero muy alta de calcio, oxígeno y fósforo (Espinoza J., Gonzalez L. \& Ruiz P., 2013)

Para tal efecto la arginina, el bicarbonato y el carbonato de calcio, interaccionan generando una obstrucción física de los túbulos dentinarios expuesto, logrando el alivio del paciente con hipersensibilidad dentinaria. Al cepillarse, en el dentífrico ya contiene arginina, en conjunto con la saliva del paciente, reaccionarían con el bicarbonato y el carbonato de calcio, formando una estructura de bicarbonato de arginina el cual es altamente soluble. Esta estructura posee propiedades adhesivas por lo que forman una mezcla que no solo obstruiría los túbulos dentinarios abiertos, también se une a las paredes de los tubulillos, propiedad otorgada debido a la alcalinidad de la molécula (Márquez, Quintero, Sanz, Ramírez, Inostroza \& Chaparro., 2016) 


\section{Efectividad de la arginina en el tratamiento de la sensibilidad dentaria}

Vol. 2, núm. 3., (2018)

José Santiago Insuasti Rincones; Jossue Tarquino Narvaez Guerrero; Vanessa Angelica Chiquito Cedeño; Samuel Wilfrido Prado Moncada

El fin de esta investigación fue evaluar el efecto de la arginina al $8 \%$ contenido en un dentífrico, en la reducción de la hipersensibilidad dentinaria aplicados a un grupo de 30 pacientes que presentaron sensibilidad dentinaria como consecuencia del tratamiento periodontal, el cual fue valorado 4 semanas posterior de la aplicación diaria mediante el cepillado dental correcto en pacientes que acuden a las Clínicas de la Universidad de Guayaquil de la Facultad Piloto de Odontología.

\section{Metodología.}

Diseño y tipo de la investigación

Esta investigación es de tipo Cualitativa ya que en la descripción se refleja las características positivas y negativas en este tratamiento. Y Cuantitativo porque lo reflejamos en números y porcentajes de acuerdo a la respuesta del tratamiento.

También es de tipo descriptivo ya que se describe el efecto y formas de presentación de la arginina al $8 \%$ frente la presencia de sensibilidad dental en pacientes tratados periodontalmente.

Transversal porque es un estudio realizado en un mes realizado en la clínica integral.

No Experimental porque el estudio se realizó a base de observaciones de hechos que ya se han presentado y que se han manifestado en una serie de eventos y a partir de las observaciones procedimos a diseñar nuestros objetivos. 


\section{Efectividad de la arginina en el tratamiento de la sensibilidad dentaria}

Vol. 2, núm. 3., (2018)

José Santiago Insuasti Rincones; Jossue Tarquino Narvaez Guerrero; Vanessa Angelica Chiquito Cedeño; Samuel Wilfrido Prado Moncada

Es de campo ya que la investigación se la realiza en la clínica integral de la Facultad de Odontología de la ciudad de Guayaquil.

Población y muestra

La población no probabilística que sirvió como objeto de investigación fueron los pacientes que asistieron a la clínica integral de la Facultad de odontología, a realizarse un tratamiento periodontal.

En esta investigación se realizó una encuesta y se seleccionaron 30 pacientes de forma aleatoria, para poder conocer la efectividad de la arginina en los pacientes con sensibilidad dental. Muestra de intrusión fueron atendidos en la clínica de la facultad y aprobaron el conocimiento informado.

Métodos, técnicas e instrumentos

Método Científico Se estudió el desarrollo de la importancia del tema: Efectividad de la Arginina en el Tratamiento de la Sensibilidad Dentaria.

Método estadístico se realizó encuestas a los pacientes para medir el grado de sensibilidad y una reevaluación luego de la aplicación de la arginina.

Analítico-sintético Nos facilitó mucho haber analizado la información encontrado en artículos, páginas de internet, las cuales describen la problemática sobre el tema que hemos planteado en la investigación. También se consiguió profundizar en las conclusiones a la que 


\section{Efectividad de la arginina en el tratamiento de la sensibilidad dentaria}

Vol. 2, núm. 3., (2018)

José Santiago Insuasti Rincones; Jossue Tarquino Narvaez Guerrero; Vanessa Angelica Chiquito Cedeño; Samuel Wilfrido Prado Moncada

llegamos y poder ampliar nuestros conocimientos sobre el tema que es, Efectividad de la Arginina en el Tratamiento de la Sensibilidad Dentaria.

Inductivo-deductivo Toda la información de las páginas de internet se analizaron muy bien, a través de la inducción analítica, para el desarrollo del tema investigado, para esto se partió con la realización de una encuesta y la opinión de muchos autores.

La información se recolecto a través de libros, artículos científicos y test del nivel de sensibilidad dentinaria y los factores de riesgos que presentaba cada paciente.

\section{Procedimiento de la investigación}

\section{Fase I Planeación}

En esta etapa es donde elegimos nuestro tema que es Efectividad de la Arginina en el Tratamiento de la Sensibilidad Dentaria. En esta investigación se elaboró encuestas dirigidas a los pacientes que asistieron a la clínica integral de la Facultad de Odontología en los últimos meses de estudio.

\section{Fase II Ejecución}

Se encuesto a 30 pacientes en la clínica integral de la Facultad Piloto de Odontología, las preguntas fueron conforme a la escala de liker con las consideraciones muy bajo, bajo, medio, alto y muy alto. Los datos obtenidos en la encuesta se asentaron en una hoja de cálculo Excel, donde los resultados lo tabulamos en base a una distribución de frecuencias y porcentajes, en relación con los objetivos planteados. 


\section{Efectividad de la arginina en el tratamiento de la sensibilidad dentaria}

Vol. 2, núm. 3., (2018)

José Santiago Insuasti Rincones; Jossue Tarquino Narvaez Guerrero; Vanessa Angelica Chiquito Cedeño; Samuel Wilfrido Prado Moncada

Para medir el dolor de utilizo la escala Analógica Visual (EVA) esta escala consiste en una línea de $10 \mathrm{~cm}$. con un extremo marcado con "no dolor" y otro extremo que indica "el peor dolor imaginable”. El paciente marca en la línea el punto que mejor describe la intensidad de su dolor.

Fase III Comunicación de resultados

Los resultados que logramos gracias a la encuesta fueron muy satisfactorios, porque así podemos incentivar a otros estudiantes a realizar trabajos similares a nuestro tema.

Se debe considerar que en este trabajo de investigación hemos conseguido saber si la arginina es un buen tratamiento contra la sensibilidad dental.

\section{Resultados.}

De acuerdo a la encuesta los resultados se los evaluara de manera estadística, en cuadros y gráficos.

Tabla 1.- ¿Se cepilla en forma agresiva los dientes?

\begin{tabular}{|c|c|c|}
\hline $\begin{array}{c}\text { CEPILLADO } \\
\text { AGRESIVO }\end{array}$ & FRECUENCIA & PORCENTAJE \\
\hline SI & 21 & $72 \%$ \\
\hline NO & 9 & $28 \%$ \\
\hline TOTAL & 30 & $100 \%$ \\
\hline
\end{tabular}

Del total de la muestra de 30 pacientes con sensibilidad se encontró 21 con cepillado agresivo (72\%) y 9 casos sin cepillado agresivo (28\%). 


\section{Efectividad de la arginina en el tratamiento de la sensibilidad dentaria}

Vol. 2, núm. 3., (2018)

José Santiago Insuasti Rincones; Jossue Tarquino Narvaez Guerrero; Vanessa Angelica Chiquito Cedeño; Samuel Wilfrido Prado Moncada

Tabla 2.- ¿Siente dolor en los dientes cuando ingiere alimentos muy fríos o muy calientes?

\begin{tabular}{|c|c|c|}
\hline \multicolumn{3}{|c|}{ SENSIBILIDAD EN LOS DIENTES } \\
\hline ESCALA & $\mathbf{N}^{*}$ & $\%$ \\
\hline MUY BAJO & 7 & 23 \\
\hline BAJO & 20 & 67 \\
\hline MEDIO & 3 & 10 \\
\hline ALTO & 0 & 0 \\
\hline MUY ALTO & 0 & 0 \\
\hline TOTAL & 30 & 100 \\
\hline
\end{tabular}

En esta pregunta podemos observar que entre los pacientes encuestados el $67 \%$ presenta sensibilidad con los alimentos fríos o calientes el 23\%presenta una sensación alta, y un $16 \%$ una sensación de la sensibilidad a los alimentos fríos o calientes.

Tabla 3.- ¿Se ha realizado blanqueamientos dentales por el Odontólogo?

\begin{tabular}{|l|c|c|}
\hline \multicolumn{3}{|c|}{ BLANQUEAMIENTOS DENTALES } \\
\hline SI & 3 & $2 \%$ \\
\hline NO & 27 & $98 \%$ \\
\hline TOTAL & 30 & $100 \%$ \\
\hline
\end{tabular}

De los pacientes encuestados solo un $2 \%$ se han realizado blanqueamiento dentales, el 98\% desconoce de ese tipo de tratamiento. 
Efectividad de la arginina en el tratamiento de la sensibilidad dentaria

Vol. 2, núm. 3., (2018)

José Santiago Insuasti Rincones; Jossue Tarquino Narvaez Guerrero; Vanessa Angelica Chiquito Cedeño; Samuel Wilfrido Prado Moncada

Tabla 4.- ¿Cuál de las siguientes de las siguientes pasta dentales utiliza diariamente?

\begin{tabular}{|c|c|c|}
\hline CREMAS DENTALES & $\mathrm{N}^{*}$ & $\% \mathrm{i}$ \\
\hline & 0 & 0 \\
\hline \multirow[t]{2}{*}{ 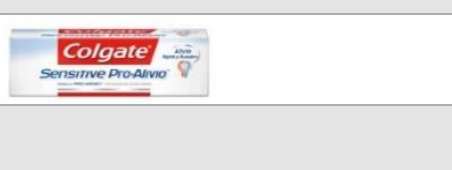 } & 0 & 0 \\
\hline & 0 & 0 \\
\hline \multirow[t]{2}{*}{ Colgate } & 30 & 100 \\
\hline & 0 & 0 \\
\hline Colgate & 0 & 0 \\
\hline TOTAL & 30 & 100 \\
\hline
\end{tabular}

Al preguntarles que tipo de pasta dental utiliza para prevenir la sensibilidad, entre las distintas mascar comerciales que existe el 100\% utiliza la pasta dental Colgate.

Tabla 5.- ¿Recibió usted alguna vez tratamiento para la sensibilidad dentaria?

\begin{tabular}{|l|c|c|}
\hline \multicolumn{3}{|c|}{ TRATAMIENTO PARA LA } \\
SENSIBILIDAD DENTARIA \\
\hline NO & 18 & $60 \%$ \\
\hline SI & 12 & $40 \%$ \\
\hline TOTAL & 30 & $100 \%$ \\
\hline
\end{tabular}

El 60\% manifiestan que NO recibió tratamientos para la sensibilidad dental y el $40 \%$ que SI recibió tratamiento. 


\section{Efectividad de la arginina en el tratamiento de la sensibilidad dentaria}

Vol. 2, núm. 3., (2018)

José Santiago Insuasti Rincones; Jossue Tarquino Narvaez Guerrero; Vanessa Angelica Chiquito Cedeño; Samuel Wilfrido Prado Moncada

Después de la encuesta se procede a realizar la prueba de sensibilidad con la escala de EVA la cual se conoce que se mide el dolor en una línea del 1 al 10.

\begin{tabular}{|c|c|c|c|c|c|c|}
\hline \multirow{2}{*}{$\begin{array}{c}\text { ESCALA DE } \\
\text { EVA }\end{array}$} & \multicolumn{2}{|c|}{ MASCULINO } & \multicolumn{2}{|c|}{ FEMENINO } & \multicolumn{2}{|c|}{ TOTAL } \\
\cline { 2 - 7 } & $\mathrm{N}^{*}$ & $\%$ & $\mathrm{~N}^{*}$ & $\%$ & $\mathrm{~N}^{*}$ & $\%$ \\
\hline $\begin{array}{c}\text { AUSENCIA DE } \\
\text { SENSIBILIDAD }\end{array}$ & 6 & 20 & 8 & 26 & 14 & 47 \\
\hline LEVE & & & & & & \\
\hline MODERADA & 2 & 20 & 6 & 20 & 12 & 40 \\
\hline INTENSA & 0 & 0 & 2 & 7 & 4 & 13 \\
\hline TOTAL & 14 & 47 & 16 & 53 & 30 & 100 \\
\hline
\end{tabular}

\begin{tabular}{|c|c|c|}
\hline SEXO & N* & \% \\
\hline Masculino & 14 & 47 \\
\hline femenino & 16 & 53 \\
\hline total & 30 & 100 \\
\hline
\end{tabular}

\begin{tabular}{|c|c|c|}
\hline \multirow{2}{*}{ ESCALA DE EVA } & \multicolumn{2}{|c|}{ TOTAL } \\
\cline { 2 - 3 } & $\mathrm{N}^{*}$ & $\%$ \\
\hline AUSENCIA DE & 0 & 0 \\
\hline SENSIBILIDAD & & \\
\hline LEVE & 4 & 13 \\
\hline MODERADA & 12 & 40 \\
\hline INTENSA & 14 & 47 \\
\hline TOTAL & 30 & 100 \\
\hline
\end{tabular}

Observamos que el $47 \%$ de los pacientes presenta intensa sensibilidad dental, el $40 \%$ presenta una sensibilidad moderada y un $13 \%$ presenta una sensibilidad leve. 
Efectividad de la arginina en el tratamiento de la sensibilidad dentaria

Vol. 2, núm. 3., (2018)

José Santiago Insuasti Rincones; Jossue Tarquino Narvaez Guerrero; Vanessa Angelica Chiquito

Cedeño; Samuel Wilfrido Prado Moncada

\section{REEVALUACIÓN}

Segundo Objetivo Específico: Determinar el efecto desensibilizante de la arginina aplicada a los pacientes.

Después de 30 días se realizó la reevaluación para comprobar si los hábitos del mal cepillado y la sensibilidad disminuyo.

Tabla 6.- ¿Mejoro su técnica de cepillado después del tratamiento?

\begin{tabular}{|c|c|c|}
\hline \multicolumn{3}{|c|}{ MEJORO SU TÉCNICA DE } \\
CEPILLADO DESPUÉS DEL \\
TRATAMIENTO \\
\hline NO & 3 & $2 \%$ \\
\hline SI & 27 & $98 \%$ \\
\hline TOTAL & 30 & $100 \%$ \\
\hline
\end{tabular}

En la reevaluación comprobamos que el $98 \%$ de los pacientes comenzó a mejorar su técnica de cepillado y un $2 \%$ aun no aplica las técnicas de cepillado.

Tabla 7.- ¿Persiste el dolor cuando ingiere alimentos muy fríos o muy calientes?

\begin{tabular}{|c|c|c|}
\hline \multicolumn{3}{|c|}{ SENSIBILIDAD EN LOS DIENTES } \\
\hline ESCALA & $\mathrm{N}^{*}$ & $\%$ \\
\hline MUY BAJO & 28 & 98 \\
\hline BAJO & 2 & 2 \\
\hline MEDIO & 0 & 0 \\
\hline ALTO & 0 & 0 \\
\hline MUY ALTO & 0 & 0 \\
\hline TOTAL & 30 & 100 \\
\hline
\end{tabular}




\section{Efectividad de la arginina en el tratamiento de la sensibilidad dentaria}

Vol. 2, núm. 3., (2018)

José Santiago Insuasti Rincones; Jossue Tarquino Narvaez Guerrero; Vanessa Angelica Chiquito Cedeño; Samuel Wilfrido Prado Moncada

El 98\% refiere que ya no presenta dolor frente al consumo de alimentos fríos o calientes un $2 \%$ indica que muy poco le afecta el frio o lo caliente.

Tabla 8.- ¿Presenta sensibilidad en los dientes actualmente aplicando la escala EVA?

\begin{tabular}{|c|c|c|c|c|c|c|}
\hline \multirow{2}{*}{$\begin{array}{c}\text { ESCALA DE } \\
\text { EVA }\end{array}$} & \multicolumn{2}{|c|}{ MASCULINO } & \multicolumn{2}{|c|}{ FEMENINO } & \multicolumn{2}{|c|}{ TOTAL } \\
\cline { 2 - 7 } & $\mathrm{N}^{*}$ & $\%$ & $\mathrm{~N}^{*}$ & $\%$ & $\mathrm{~N}^{*}$ & $\%$ \\
\hline $\begin{array}{c}\text { AUSENCIA DE } \\
\text { SENSIBILIDAD }\end{array}$ & 13 & 40 & 16 & 53 & 21 & 88 \\
\hline LEVE & 2 & 7 & 0 & 0 & 9 & 12 \\
\hline MODERADA & 0 & 0 & 0 & 0 & 0 & 0 \\
\hline INTENSA & 0 & 0 & 0 & 0 & 0 & 0 \\
\hline TOTAL & 14 & 47 & 16 & 53 & 30 & 100 \\
\hline
\end{tabular}

En la reevaluación con la prueba EVA nos damos cuenta que el $88 \%$ de los paciente presenta ausencia de la sensibilidad dental y un $12 \%$ presenta una sensación leve.

Tercer objetivo específico: Establecer el tipo de tratamiento para la sensibilidad dental de los pacientes. 
Efectividad de la arginina en el tratamiento de la sensibilidad dentaria

Vol. 2, núm. 3., (2018) José Santiago Insuasti Rincones; Jossue Tarquino Narvaez Guerrero; Vanessa Angelica Chiquito Cedeño; Samuel Wilfrido Prado Moncada

Tabla 9.- ¿Qué producto uso para su tratamiento de sensibilidad?

\begin{tabular}{|c|c|c|}
\hline CREMAS DENTALES & $\mathbf{N}^{*}$ & $\% \mathrm{i}$ \\
\hline & 0 & 0 \\
\hline \multirow{2}{*}{ Colgate $=9$} & 0 & 0 \\
\hline & 0 & 0 \\
\hline \multirow{2}{*}{ 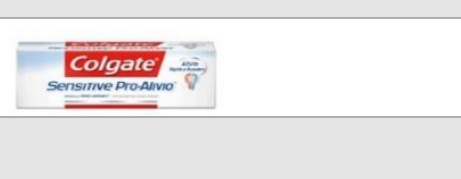 } & 30 & 100 \\
\hline & 0 & 0 \\
\hline Colgate & 0 & 0 \\
\hline TOTAL & 30 & 100 \\
\hline
\end{tabular}

Los pacientes que presentaban sensibilidad dental, entre las distintas mascar comerciales que existe el $100 \%$ utilizo en el tratamiento Colgate sinsitive.

Tabla 10.- Los pacientes fueron divididos en dos grupos por sexo 14 hombres y 16 mujeres

\begin{tabular}{|c|c|c|}
\hline SEXO & $\mathbf{N}^{*}$ & $\%$ \\
\hline Masculino & 14 & 47 \\
\hline femenino & 16 & 53 \\
\hline total & 30 & 100 \\
\hline
\end{tabular}




\section{Efectividad de la arginina en el tratamiento de la sensibilidad dentaria}

Vol. 2, núm. 3., (2018)

José Santiago Insuasti Rincones; Jossue Tarquino Narvaez Guerrero; Vanessa Angelica Chiquito Cedeño; Samuel Wilfrido Prado Moncada

\section{Discusión.}

Se determina que uno de los factores que ocasiono la sensibilidad dental en los pacientes fue por el cepillado brusco el $72 \%$ no tiene conocimiento sobre las técnicas adecuadas del cepillado, lo que este trabajo tiene coincidencia con un estudio realizado por (NINALAYA URBINA, 2011) donde en su estudio en la que analizo 100 pacientes para comprobar que ocasionaba la sensibilidad dental en los pacientes seleccionados, en ese estudio se clasifico de la siguiente manera, por: presencia de microorganismos del biofilm dental $16 \%$, acción mecánica del cepillado $54 \%$, fuerzas oclusales $30 \%$.

Al determinar la reducción de la hipersensibilidad dentinaria con el dentífrico que contiene arginina $8 \%$ podemos observar que hubo una disminución promedio del dolor de acuerdo a la prueba EVA se establece que $88 \%$ de los paciente presenta ausencia de la sensibilidad dental y un $12 \%$ presenta una sensación leve. A pesar de que en este estudio se demostró que hubo una disminución de la sensibilidad con este agente terapéutico, (Márquez, Quintero, Sanz, \& Ramírez, 2011), en su estudio realizado donde se comparó el efecto de la arginina $8 \%$ - carbonato de calcio y del fluoruro de sodio al 5\% en la reducción de la hipersensibilidad dentinaria posterior a la terapia periodontal, se observó una mayor eficacia en la reducción del dolor en el grupo que utilizó arginina al 8\% y carbonato de calcio en comparación con la aplicación de barniz de fluoruro de sodio al 5\%.

En nuestro estudio se utilizó una crema dental que contiene arginina la cual se les facilito a los pacientes y se les dio las indicaciones de cómo utilizarla con una técnica de cepillado correcta, y el resultado del tratamiento fue efectivo de un $88 \%$. En estudios realizados por Schiff 


\section{Efectividad de la arginina en el tratamiento de la sensibilidad dentaria}

Vol. 2, núm. 3., (2018)

José Santiago Insuasti Rincones; Jossue Tarquino Narvaez Guerrero; Vanessa Angelica Chiquito Cedeño; Samuel Wilfrido Prado Moncada

en el 2010, en el cual se evaluó clínicamente la eficacia de la pasta desensibilizante que contiene arginina $8 \%$ - carbonato de calcio en proveer un alivio instantáneo y duradero en pacientes con hipersensibilidad, se demostró que provee una reducción estadísticamente significativa en la disminución de la hipersensibilidad dentinaria después de aplicar el producto en forma inmediata, y que ésta se mantuvo por un periodo de 28 días.

Por lo tanto, según los estudios mencionados anteriormente, se establece que el dentífrico que contiene arginina al $8 \%$ - carbonato de calcio, es superior a los otros agentes desensibilizantes utilizados, lo que no concuerda con los datos obtenidos en este trabajo de investigación, en el cual no hubo una diferencia estadísticamente significativa entre los dentríficos estudiados.

Es decir que la arginina para fortalecer su efecto contra la sensibilidad dental tendremos que combinarla con otras sustancias.

\section{Conclusiones.}

El uso de los dentífricos que contienen arginina $8 \%$ para la reducción de la hipersensibilidad dentinaria tras una y tres semanas de uso cumplen satisfactoriamente con los resultados deseados ya que disminuye la sensibilidad dental.

Tanto los dentífricos que contienen arginina $8 \%$ son efectivos en la reducción de la hipersensibilidad dentinaria tras una y tres semanas de uso. 


\section{Efectividad de la arginina en el tratamiento de la sensibilidad dentaria}

Vol. 2, núm. 3., (2018)

José Santiago Insuasti Rincones; Jossue Tarquino Narvaez Guerrero; Vanessa Angelica Chiquito Cedeño; Samuel Wilfrido Prado Moncada

A pesar de que los resultados son positivos en relación a la disminución de la sensibilidad, se necesitaría obtener un mayor tamaño de la muestra, y además prolongar el tiempo de uso del agente terapéutico para hacerlos comparables.

Una de las limitaciones que podría haber influenciado en el presente estudio, es que no fue posible tener certeza del cumplimiento de las indicaciones por parte de los pacientes que utilizaron los dentífricos.

Se sugiere en investigaciones futuras que se considere un tiempo mayor de tratamiento e incluir más pastas dentales para que con cada pasta dental se pueda observar si hay diferencias estadísticamente significativas entre los dentífricos que contienen arginina $8 \%$ y carbonato de calcio.

Realizar un estudio tipo doble ciego y longitudinal con el objetivo de que los pacientes desconozcan el tipo de tratamiento utilizado, disminuyendo la posibilidad de que existan sesgos, para poder lograr una mayor precisión de los resultados.

\section{Bibliografía.}

Alvarez, Arroyo, Aranguiz, Contreras, \& Moncada. (julio de 2010). Diagnóstico y Tratamiento de la Hipersensibilidad. Revista Dental de Chile, 101(3), 17-25.

Ardila, M. (11 de septiembre de 2009). Hipersensibilidad dentinal: Una revisión de su etiolgia, patogenesis y tratamiento. AVANCES EN ODONTOESTOMATOLOGÍA, 25(3), 137-146. Obtenido de scielo.isciii.es: http://scielo.isciii.es/pdf/odonto/v25n3/original2.pdf

Espinoza J., Gonzalez L. \& Ruiz P. (2013). Tratamiento de la hipersensibilidad dentinaria post terapia periodontal, mediante el uso de dos dentífricos desensibilizantes. Rev. Clin. Periodoncia Implantol. Rehabil. Oral, 6(2), 78-82. 


\section{Efectividad de la arginina en el tratamiento de la sensibilidad dentaria}

Vol. 2, núm. 3., (2018)

José Santiago Insuasti Rincones; Jossue Tarquino Narvaez Guerrero; Vanessa Angelica Chiquito Cedeño; Samuel Wilfrido Prado Moncada

Márquez, M., Quintero, A., Sanz, A., \& Ramírez, V. (2011). Efecto de la arginina 8\%-carbonato de calcio y del fluoruro de sodio al $5 \%$ en la reducción de la hipersensibilidad dentinaria post terapia periodontal: ensayo clínico. Revista clínica de periodoncia, implantología y rehabilitación oral, 4(1), 22-25.

Márquez, Quintero, Sanz, Ramírez, Inostroza \& Chaparro. (25 de abril de 2016). ANÁLISIS IN VITRO DE TRES DENTÍFRICOS CON AGENTES ANTIBACTERIANOS Y SU EFICACIA FRENTE A STREPTOCOCCUS MUTANS (ATCC 25175) $Y$ LACTOBACILLUS ACIDOPHILUS (ATCC 4356). Quito: Universidad Central del Ecuador.

NINALAYA URBINA, A. (2011). “HIPERSENSIBILIDAD DENTINARIA PRIMARIA. Lima: UNIVERSIDAD PERUANA CAYETANO HEREDIA. 